\title{
Plädoyer für Bologna? Justizminister im Kreuzverhör
}

\author{
Hearing des Stifterverbands für die Deutsche Wissenschaft
}

13. Februar 2008, Bonn

Im Bonner Wissenschaftszentrum drehte sich am 13. Februar bei dem vom Stifterverband für die Deutsche Wissenschaft initiierten Hearing „Neue Wege in der Juristenausbildung “ alles um die Frage, welches Mittel zur Lösung der seit Jahren beklagten Missstände die wenigsten Risiken und Nebenwirkungen, insbesondere für die angehenden Juristinnen und auf die hohe Qualität der Ausbildung hierzulande, in sich birgt. Die beiden Vertreterinnen der Mitglieder in Ausbildung im djb, Anna Eberhard und Barbara Dittmann, die für den Bundesvorstand an dem Hearing teilnahmen, konnten sich einen Überblick über einige der wichtigsten Reformvorschläge verschaffen, die vom „Mannheimer Modell“ über das „Stuttgarter Modell“ zum „NRW-Modell“ reichen und unterschiedlichste Lösungsansätze bieten. Der Unterschied liegt überwiegend im Detail. Denn durchweg bieten die vorgestellten Modelle vernünftige Ansätze, wie die Ausbildungsordnung den notwendigen Reformen unterzogen werden und dennoch den tradierten hohen Standard beibehalten kann.

Zur Begrüßung wies Prof. Dr. Barbara DaunerLieb, Richterin am Verfassungsgerichtshof für das Land Nordrhein-Westfalen und Direktorin des Instituts für Gesellschaftsrecht, Leiterin der Abt. 3: Europäisches Gesellschaftsrecht an der Universität Köln, auf das typisch deutsche Ausbildungsmerkmal der „Wissenschaftlichkeit“ hin, die Verzahnung von Theorie und Praxis, wie sie so im Ausland nicht gesehen wird. Einig waren sich im Anschluss auch die anderen Referenten, dass die hohe Qualität der deutschen Ausbildung trotz der Verbesserungsbedürftigkeit im Einzelnen keinem vermeintlichen Neuerungszwang zum Opfer fallen dürfe. So hält das von der Justizministerin des Landes Nordrhein-Westfalen, Roswitha MüllerPiepenkötter, vorgestellte „NRW-Modell“ ebenso wie das „Stuttgarter Modell“ neben allen notwendigen Veränderungen am Modell des Einheitsjuristen als Qualitätsmerkmal fest. Weitgehende Einigkeit herrschte auch hinsichtlich der Notwendigkeit der Beibehaltung (zumindest) eines Staatsexamens.

\section{Die einzelnen Modelle im Überblick}

Die Bachelor- und Master-Modelle wurden anhand eines Fragenkatalogs vorgestellt und diskutiert.

Den Referenten wurden die folgenden acht Fragen gestellt, zu denen sie in ihren Vorträgen und in der anschließenden Diskussion Stellung nahmen:

1. Müssen wir Ausbildungsfragen ernster nehmen?

2. Muss die juristische Didaktik in Deutschland eine andere Bedeutung bekommen als bisher?

3. Soll am „Einheitsjuristen“ festgehalten werden?

4. Ist mehr Durchlässigkeit wünschenswert? Wie kann sie erreicht werden?

5. Kann sichergestellt werden und wenn ja, wie, dass der Bachelor erfolgreich sein wird? Mit anderen Worten: ist der Bachelor marktkonform?

6. Wie ist dem „Massenphänomen“ an den Universitäten zu begegnen?

7. Wie kann gewährleistet werden, dass der Bachelor auch bereits qualifizierend ist?

8. Wie kann gewährleistet werden, dass der Arbeitsmarkt auch bereit für den Bachelor ist?

Das ,3+2-Modell“ der nordrhein-westfälischen Justizministerin Roswitha Müller-Piepenkötter spricht sich für mehr Durchlässigkeit in der Ausbildung aus. An ein dreijähriges intensives Studium in Kerngebieten, in denen solides juristisches Grundwissen und Methodik vermittelt wird (= Mindeststandard) und das mit der Bachelor-Arbeit abschließt, soll sich eine staatliche Eignungsprüfung anschließen, nach der bei Eignung der juristische Vorbereitungsdienst angetreten wird. Während bereits im Bachelor-Studium zu dem Mindeststandard 30 Prozent universitäre Schwerpunkte gesetzt werden, was der Profilstärkung der Universitäten dienen kann, sollen aus dem staatlichen Vorbereitungsdienst einzelne Teile wie beispielsweise das Zwangsvollstreckungsrecht in die Master-Phase ausgelagert werden. Denkbar ist demnach auch ein MasterStudium in Rechtspflege zur zielgenaueren Spezialisierung. Ein Bachelor ist nach diesem Modell bereits nach sechs Semestern möglich, für die ein Markt bereits bestehe.
Barbara Dittmann,

LL.M.

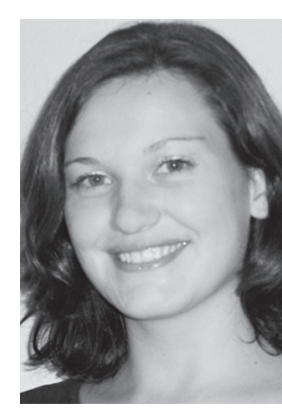

Vertreterin der Mit-

glieder in Ausbildung im Bundesvorstand des djb; Rechtsreferendarin, Dortmund 
Das „Stuttgarter Reformmodell“, gemeinsam von den Justizministern Prof. Dr. Ulrich Goll (Baden-Württemberg) und Gert Mackenroth (Sachsen) entwickelt, will eine „echte Strukturreform “ herbeiführen. Im ersten bis vierten Semester soll ein Grundlagenstudium angeboten werden, welches durch Praktika zu ergänzen ist. Dieses Grundlagenstudium soll ebenso wie die beiden sich anschließenden Vertiefungssemester neben Vorlesungen überwiegend in Kleingruppen stattfinden. In die Abschlussnote sollen die Studienleistungen und die Bachelor-Arbeit einfließen. Getreu dem Motto „Wer lehrt, prüft! “ würden hier institutionelle Ausbilder zurückgedrängt. Im anschließenden Praxisjahr, welches von Vertretern der juristischen Professionen geleitet wird, wird die Lehre parallel zugelassen. An die Praxis schließt sich dann eine einjährige Vertiefungs- und Wahlfachphase an, in der das gewählte Spezialgebiet wissenschaftlich vertieft wird und die mit der universitären Prüfung und Master-Arbeit unter staatlicher Beteiligung (Praktiker als Korrektoren, Supervision durch das jeweilige LJPA) abgeschlossen wird. Im Anschluss an dieses fünfjährige praxisorientierte Studium folgt die einjährige Berufseinarbeitungsphase ohne abschließende Prüfung, für die eine Intensivierung der Betreuung gefordert wird. Die Vertreter dieses Modells sind optimistisch, dass die Verhältnisse sich trotz übergangsweise schlechter Arbeitsmarktchancen für Absolventen schnell anpassen werden.

Der Vorschlag von Harald Schliemann' und Prof. Dr. Horst Konzen dagegen will der Tendenz, Schwerpunkte fortzubilden, entgegenwirken. Eine Stoffreduzierung, zum Beispiel die Konzentration auf das BGB, bringe mehr, da exemplarisches Lernen dazu befähige, sich auch in andere Fächer einzuarbeiten. Ihr Modell sieht eine 2-Stufen-Ausbildung nach der Quotierung „4+x“ vor. In der ersten Stufe findet eine einheitliche Ausbildung statt. Die Einheitsausbildung sieht auch Prof. Konzen als notwendig an; der Wechsel vom Anwalt zum Richter und umgekehrt müsse unbedingt gewährleistet sein. Ein sich anschließender Master soll keine Zulassungsvoraussetzung für die Ausübung von Kernberufen sein, sondern eine reine Zusatzqualifikation bieten (z.B. im Bereich Steuern oder BWL). Eine Spartenausbildung wie beispielsweise nach den Vorgaben des DAV halten die Vertreter dieses Modells wegen der ähnlichen Anforderungen und Inhalte grundsätzlich für sinnvoll, jedoch nur unter weitgehender Wahrung des Modells „Einheitsjurist“ und der erforderlichen Durchlässigkeit der einzelnen Berufe. Das Leitmotiv „Qualität statt Quantität“ verbiete die Reduzierung der Ausbildungsdauer zum Selbstzweck; vielmehr müsse sich die Zeitdauer am Inhalt der Ausbildung orientieren.

Das „4-Stufen-Modell“ des Hamburger Notars Dr. Jens Jeep fordert: Mehr Eigenverantwortung der Universitäten. Durch die „Entkoppelung des Jurastudiums vom Staatsexamen " werde gewährleistet, dass die Universitäten tatsächlich zum Staatsexamen ausbilden. Erforderlich seien Statistiken der einzelnen Universitäten zu den Bachelor- und Examensnoten, die eine Art Benchmarking zwischen den einzelnen
Universitäten ermöglichen. Die Qualität der Ausbildung wird demnach durch ein höheres Maß an Wissenschaftlichkeit der Universitäten gewährleistet, wozu der Wettbewerb zwischen den Ausbildungsstandorten zwangsläufig führe. Nach dem vierjährigen Grundlagenstudium, das mit dem Bachelor-Abschluss auf der Grundlage studienbegleitender Prüfungen endet und das zu 30 Prozent individuell gewählte Praktika beinhaltet, schließt sich entweder nach Absolvierung eines einheitlichen Staatsexamens das einjährige Referendariat oder ein individuelles Master-Studium als Zusatzausbildung an. Angedacht ist nach diesem Modell, die Zulassung zum Staatsexamen von der Bachelor-Note „befriedigend“ abhängig zu machen. Zwar sei eine Vereinbarkeit mit Art. 12 GG zunächst fraglich, jedoch sei auch heute für Volljuristen die „Befähigung zum Richteramt“ nicht flächendeckend gegeben. Besser sei es, stattdessen einen qualitativ hochwertigen Master, beispielsweise auch einen Rechtspflege-Bachelor für Notariatsangestellte, anzubieten, da der Markt für verschiedene Ansprüche verschiedene Absolventen erfordere.

Das „Mannheimer Modell“ spricht sich für einen „gestuften Kombinationsstudiengang“ aus. Demnach müssen auch Wirtschaftsrecht und BWL im Bachelor-Studium auf hohem Niveau angeboten werden, da sich an den Gegebenheiten des Arbeitsmarktes zeige, dass auch von Juristen ein erweiterter Sachverstand in spezifischen Bereichen erforderlich sei. Wissenschaftlich fundierte Studiengänge und eine Generalistenausbildung sind demnach auch für nicht-juristische Berufe erforderlich, jedoch müssten umgekehrt auch die neueren berufsspezifischen Anforderungen, zu denen weiterhin auch Finanzen und M\&A zählten, die ein erhebliches Arbeitsmarktpotenzial bildeten, mit einbezogen werden. Nach dem „gestuften Kombinationsstudiengang“ könnten Strafrecht und Öffentliches Recht in einem Ergänzungsstudium nachgeholt werden.

\section{Die Arbeit des djb zum Thema Ausbildung}

Auf diese (s. S. 101) und weitere Fragen eine Antwort zu finden, hat sich auch der djb mit einer jetzt (wieder) eingerichteten Arbeitsgruppe „Bologna“ zur Aufgabe gemacht. Die Arbeitsgruppe wird dabei an die von ihrer Vorgängerin, dem Arbeitsstab „Ausbildungsreform“, erarbeiteten Standpunkte anknüpfen. In der aktuellen Diskussion spiegelt sich vieles von dem wider, was bereits 1998 vom djb in Form von neun Thesen erarbeitet und als änderungsbedürftig erkannt wurde. Geändert haben sich in den letzten zehn Jahren aber vor allem die Rahmenbedingungen:

- der Bologna-Prozess und die darin geforderte europaweite Umstellung auf ein Bachelor/Master-System,

- die zunehmend internationale Ausrichtung von Unternehmen und Kanzleien,

- die europäische und internationale Konkurrenz,

1 Harald Schliemann war bis Mai 2008 Thüringer Justizminister. 
- zahlreiche für Juristen neu erschlossene Tätigkeitsfelder, womit nicht zuletzt höhere und über die juristischen Kernkompetenzen hinausreichende Anforderungen an den juristischen Nachwuchs gestellt werden.

Die djb-Arbeitsgruppe „Bologna“ sucht daher nach Lösungen, die den aktuellen Rahmenbedingungen gerecht werden und hat das Ziel, ein mit der deutschen Ausbildungstradition zu vereinbarendes modernes Konzept zu entwickeln. $\mathrm{Zu}$ berücksichtigen sind allem voran die Anpassung der Aus- bildung an die Berufswirklichkeit und die Befähigung der angehenden Juristinnen und Juristen, heute gefragte Tätigkeiten auszuüben sowie die Wettbewerbsfähigkeit des deutschen Ausbildungsmodells im europäischen und internationalen Vergleich.

Bis Ende Juni können sich Kolleginnen, die an einer Mitarbeit interessiert sind, per E-Mail (geschaeftsstelle@djb.de) oder postalisch (Anklamer Straße 38, 10115 Berlin) bei der Bundesgeschäftsstelle für die Teilnahme an dieser Arbeitsgruppe melden.

\section{Reform der Juristenausbildung}

\section{Neun Thesen des djb}

(Stand: 5. Juli 1998):

1. Die einheitliche Ausbildung zum sog. Einheitsjuristen soll beibehalten werden. Die bisherige breit angelegte zweistufige Ausbildung macht deutsche Juristinnen und Juristen vielseitig einsetzbar. Ihr Ruf in europäischen und in ternationalen Kreisen ist ausgezeichnet.

2. Die große Zahl der Auszubildenden in Studium und Referendariat sowie die Finanznot der öffentlichen Hand zwingen jedoch zu einer Straffung und inhaltlichen Verbesserung der Ausbildung, sind aber kein Grund, von dem Modell des Einheitsjuristen abzugehen.

3. Ziel der Ausbildung muss die Berufsbefähigung sein, nicht die Berufsfertigkeit. Die Justizlastigkeit der Ausbildung kann und muss durch veränderte Ausbildungsschwerpunkte und -inhalte korrigiert werden. Diese Berufsbefähigung erfordert neben dem Studium einen zweiten praxisbezogenen Ausbildungsabschnitt.

4. Die Reform muss bereits beim Studium ansetzen. Das Studium soll in ein Pflichtfachstudium (6 Semester) und ein Wahlfachstudium (2 Semester) gegliedert werden.

5. Zum Abschluss des 4. Semesters findet eine verbindliche Eignungs- und Erfolgskontrolle statt. Das Pflichtstudium endet mit einer Abschlussprüfung. Am Ende des Wahlfachstudiums wird in den gewählten Fächern geprüft. Die Examensnote wird aus den Ergebnissen der Pflicht- und Wahlfachprüfungen gebildet (Universitätsexamen).

6. Das Referendariat soll zwei Jahre dauern und folgende Stationen umfassen:

Zivilgerichtsbarkeit (6 Monate), Strafgerichtsbarkeit/ Staatsanwaltschaft (3 Monate), Verwaltung (4 Monate), Anwaltsstation (6 Monate) und Wahlstation (5 Monate). Nach jeder der ersten drei Stationen werden Examensklausuren geschrieben, am Ende der dritten Station findet eine mündliche Prüfung statt. Die Anwaltsstation schließt mit einer mündlichen Prüfung ab. Die Wahlstation kann in den Bereichen Justiz, Verwaltung, Anwaltschaft und Wirtschaft abgeleistet werden. Die Bewertung dieser Station erfolgt durch schriftliche Beurteilung. Die Examensnote wird ge- bildet aus den Ergebnissen der Einzelabschlüsse unter Berücksichtigung der Beurteilungen aus den Stationen und Arbeitsgemeinschaften.

Die Qualität des Referendariats kann gesteigert werden durch Fortbildungsmaßnahmen für alle Ausbildenden sowie durch veränderte Inhalte der begleitenden Arbeitsgemeinschaften (z.B. Umgang mit Parteien, Personalführung, Arbeitsorganisation, Streitschlichtung). Die inhaltliche Ausgestaltung der Anwaltsstation einschließlich der begleitenden Arbeitsgemeinschaften obliegt den Anwaltskammern.

7. Bei der Finanzierung des Studiums wird sowohl für den Staat wie auch für die Unterhaltspflichtigen eine deutliche Kostenentlastung durch die Eignungs- und Erfolgskontrolle während des Studiums und die verbindlich vorgeschriebene Höchstdauer von acht Semestern einschließlich der Prüfung eintreten. Jegliche Reform muss sicherstellen, dass die Unterhaltsaufwendungen der Eltern für studierende Kinder steuerlich angemessen berücksichtigt werden und dass die staatlichen Förderleistungen für Studierende, deren Eltern nicht in der Lage sind, ein Studium zu finanzieren, nicht weiter eingeschränkt werden.

8. Für die Ableistung des Referendariats ist ein öffentlichrechtliches Ausbildungsverhältnis dem Beamtenverhältnis auf Widerruf vorzuziehen. Nur diese Rechtsform gewährleistet eine angemessene soziale Absicherung gerade auch bei Arbeitslosigkeit nach dem Examen.

9. Die Ausbildungsvergütung muss sich in ihrer Höhe am angemessenen Lebensbedarf orientieren. Sie soll gewährleisten, dass während der Ausbildung keine Nebentätigkeit aufgenommen werden muss. Das schließt eine sozialverträgliche Herabsetzung der derzeitigen Höhe der Bezüge nicht aus. Ein weiterer Einspareffekt wird dadurch erzielt, dass unmittelbar nach der letzten Station die Examensnote errechnet werden kann und das Ausbildungsverhältnis damit endet. 